\title{
Lung angiotensin converting enzyme activity in rats with differing susceptibilities to chronic hypoxia
}

\author{
PETER JEDERLINIC, NICHOLAS S HILL, LO CHANG OU, BARRY L FANBURG \\ From the Pulmonary Division, New England Medical Center, Boston; and the Department of Physiology, \\ Dartmouth Medical School, Hanover, New Hampshire, USA
}

\begin{abstract}
The decrease in lung angiotensin converting enzyme (ACE) activity occurring in rats during chronic hypoxia might be related to the pulmonary haemodynamic response or to the hypoxia. A study in rats was carried out to investigate this question. Rats from the Hilltop (H) strain are known to develop more severe pulmonary hypertension as a result of chronic hypoxia than rats from the Madison (M) strain despite having virtually identical arterial and mixed venous oxygen tensions. Rats from $\mathbf{H}$ and $\mathrm{M}$ strains were exposed to hypoxia $(0.5 \mathrm{~atm})$ for 3-21 days and lung and serum ACE activities were determined. After three days' hypobaria lung ACE activity was significantly lower and serum ACE significantly higher in $\mathbf{H}$ than in $\mathbf{M}$ rats. Linear regressions for lung ACE activity and right ventricular:body weight ratios showed significant inverse correlations and were similar in the two strains. The results suggest that pulmonary hypertension and not hypoxia determines the reduction in lung ACE activity, possibly by releasing ACE into the blood stream.
\end{abstract}

\section{Introduction}

Angiotensin converting enzyme (ACE) transforms angiotensin I into the vasoconstrictor angiotensin II and degrades the vasodilator bradykinin. As a large portion of ACE in the body is located on the luminal surface of the pulmonary endothelium, ${ }^{\prime}$ investigators have speculated that lung ACE contributes to the regulation of pulmonary and systemic vasomotor tone and has a role in the development of pulmonary hypertension in conditions such as chronic hypoxia. ${ }^{2}$

Studies on the behaviour of lung ACE during chronic hypoxia have yielded conflicting results. Molteni et al $^{3}$ observed an increase in lung ACE activity in chronically hypoxic mice, whereas recent investigations in rats showed decreased lung ACE activity during chronic hypoxia. ${ }^{46}$ Rats with pulmonary hypertension induced by monocrotaline injection also have decreased lung ACE activity, suggesting the possibility that the haemodynamic response rather than chronic hypoxia in itself lowers lung ACE activity.

We have identified two strains of Sprague Dawley rat with very different haemodynamic responses to chronic hypoxia. ${ }^{7}$ The Hilltop $(\mathrm{H})$ strain develops

Address for reprint requests: Dr Nicholas S Hill, Pulmonary Division, Rhode Island Hospital, Providence, RI 02903, USA.

Accepted 4 May 1988 severe pulmonary hypertension, right ventricular hypertrophy, and polycythaemia after a two to four week exposure to hypobaria $(0.5 \mathrm{~atm})$, whereas the haemodynamic responses in the Madison strain are much milder. Despite these differing cardiopulmonary responses, however, the degree of hypoxaemia is similar in the two strains, as shown by nearly identical arterial and mixed venous oxygen tensions after four weeks' hypobaria. ${ }^{8}$ Responses of lung ACE activity to chronic hypoxia in these two strains might therefore provide a means of separating the effects of haemodynamic changes from those of hypoxia. If haemodynamic responses determine lung ACE activity, the levels in the $H$ and $M$ strains would be expected to differ. If lung ACE activity is primarily determined by the degree of hypoxaemia, the levels should be similar. To test these possibilities we exposed the two strains to chronic hypobaria and assayed lung ACE activity.

\section{Methods}

Sprague-Dawley rats of equivalent ages were obtained from Hilltop Laboratories, Scottsdale, Pennsylvania (altitude $H$ susceptible strain- $H$ rats) and from Harlan Sprague-Dawley, Madison, Wisconsin (M strain). $\mathrm{H}$ rats weighed $200-230 \mathrm{~g}$ and $\mathrm{M}$ rats 180 $200 \mathrm{~g}$ at the start of exposure. High altitude (HA) rats were placed in hypobaric chambers at $0.5 \mathrm{~atm}$, and sea 
level (SL) controls were placed in adjacent cages at ambient pressure. Rats had access to standard diets and unrestricted water, and were removed from hypobaria every other day for 20 minutes while cages were cleaned and food and water replenished.

Five HA rats and three SL rats from each strain were removed from their chambers after $3,7,14$ and 21 days and anaesthetised within 20 minutes with pentobarbital $30 \mathrm{mg} / \mathrm{kg}$ intraperitoneally. The animals were weighed and the right jugular vein was cannulated with a silastic catheter. Mean right ventricular peak systolic pressure (RVPP) was determined by averaging peak right ventricular pressures over 30 seconds while the rats breathed room air spontaneously. Blood was then withdrawn through the catheter for determination of packed cell volume by centrifugation; a separate sample was centrifuged for serum separation and freezing for later ACE activity measurements.

After exsanguination the left lung was removed, rinsed in sterile saline, and frozen. Hearts were dissected by the method of Fulton et al ${ }^{9}$ for determination of right ventricular and left ventricular plus septal weights. Because left ventricular septal weights tend to increase with chronic hypobaria in the $\mathrm{H}$ rats, ${ }^{10}$ right ventricular $100 \mathrm{~g}$ body weight (right ventricular weight index) was used as an indicator of right ventricular hypertrophy rather than right ventricular/ left ventricular plus septal weights.

For determination of ACE activity serum and lung samples were thawed. Lungs were weighed on an analytical balance, diced, suspended in 10 volumes of $0.02 \mathrm{M}$ phosphate buffered saline, and homogenised at $4^{\circ} \mathrm{C}$. Serum and lung ACE activity was determined as the hydrolysis rate of hippuryl-L-histidyl-L-leucine in $\mathrm{nmol} / \mathrm{l} \cdot \mathrm{min}^{-1}$ measured spectrophotometrically." Lung protein was measured in a separate aliquot of lung homogenate by the Lowry method, ${ }^{12}$ and lung $\stackrel{\vec{F}}{\rightarrow}$ ACE activity was expressed as $\mathrm{nmol} / \mathrm{min}$ per $\mathrm{mg}$ ? protein.

Data are expressed as mean with standard errors in $\frac{\bar{c}}{\frac{\omega}{\sigma}}$ parentheses. There were no changes over time in any of $\stackrel{\Phi}{\varrho}$ the measured variables in control rats and results for $\cong$ controls were pooled to obtain a single mean for each $\rightarrow$

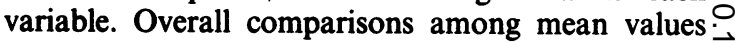
within each group of hypobaric rats over the course of $\vec{\omega}$ the study were assessed by analysis of variance. When $F$ tests gave significant results differences between $\vec{x}$ group means were tested for significance by means of ${ }^{-}$ the Student-Newman-Keuls test. ${ }^{13}$ The significance of ${ }_{0}^{\omega}$ differences between group means at each time point was assessed by $t$ tests. Linear regression analyses of $\omega$ lung ACE activity and right ventricular weight index 오 relationships were performed and $t$ tests were used for determining the significance of differences between $\mathcal{D}$ regression coefficients. Relationships between lung $\frac{\mathbb{O}}{\mathbb{P}}$ ACE activity and right ventricular peak systolic $\frac{\mathbb{D}}{3}$ pressure were analysed by means of curve fitting procedures for hyperbolic curves. ${ }^{14}$

\section{Results}

Packed cell volume and right ventricular and left ventricular plus septal weight indices rose significantly and similarly above control levels after three days' hypobaria in the two groups of rats (table). These $\stackrel{\circ}{\mathbb{D}}$ increases were enhanced by the decline in body weight $\stackrel{2}{\overrightarrow{2}}$ that occurred in both strains of rat during the first few days of hypobaric exposure. $M$ rats weighed $12 \%$ less and $\mathrm{H}$ rats $14 \%$ less after three days' hypobaria than the corresponding control rats. After the first week of hypobaria the differences between strains in packed $\vec{\partial}$ cell volume and ventricular weight indices were similar

Body and ventricular weights, packed cell volumes, and lung protein concentrations during hypobaric exposure (mean (SEM) values)

\begin{tabular}{|c|c|c|c|c|c|c|c|}
\hline Day & $n$ & $\begin{array}{l}\text { Body } \\
\text { weight }(g)\end{array}$ & $\begin{array}{l}\text { Packed cell } \\
\text { vol }(\%)\end{array}$ & $\begin{array}{l}R V P P \\
(m m H g)\end{array}$ & $\begin{array}{l}R V w t \\
\text { index } \\
(g / 100 \mathrm{~g} \mathrm{bw})\end{array}$ & $\begin{array}{l}L V+S w t \\
\text { index } \\
(g / 100 g b w)\end{array}$ & $\begin{array}{l}\text { Lung } \\
\text { homogenate } \\
\text { protein } \\
(\mathrm{mg} / \mathrm{ml})\end{array}$ \\
\hline \multicolumn{8}{|c|}{ HILLTOP } \\
\hline $0^{*}$ & 12 & $297(14)$ & $44(1)$ & $20(1)$ & $0.058(0.002)$ & $0.215(0.005)$ & 97 (5) \\
\hline 3 & 5 & $200(12) \dagger$ & $60(1) \dagger$ & $27(2) \dagger$ & $0.091(0.007) \dagger$ & $0.263(0.012) \dagger$ & $108(12)$ \\
\hline 7 & 5 & $207(13) \dagger$ & $63(1)+\S$ & $32(2)+\S$ & $0.092(0.015) \dagger$ & $0.220(0.015)$ & 99 (7) \\
\hline 14 & 5 & $241(13) \dagger$ & $59(2) \dagger$ & $44(5)+\S$ & $0.120(0.010)+\S$ & $0.245(0.023) \dagger$ & 94 (4) \\
\hline 21 & 5 & $268(15)$ & $71(3)+8$ & $53(6)+\S$ & $0.150(0.010)+\S$ & $0.270(0.027)+\S$ & $106(6)$ \\
\hline \multicolumn{8}{|c|}{ MADISON } \\
\hline $0^{*}$ & 12 & $275(14)$ & $44(1)$ & $21(1)$ & $0.055(0.002)$ & $0.224(0.004)$ & $102(6)$ \\
\hline 3 & 5 & $191(7) \dagger$ & $58(2) \dagger$ & 24 (1) & $0.097(0.007) \dagger$ & $0.286(0.011) \dagger$ & $101(6)$ \\
\hline 7 & 5 & 185 (15)† & 60 (1) $+\S$ & $27(2)+\S$ & $0.081(0.007) \dagger$ & $0.243(0.015) \dagger$ & 93 (4) \\
\hline 14 & 5 & $231(14) \dagger$ & $63(3) \dagger$ & $33(4)+\S$ & $0.083(0.011)+\S$ & $0.219(0.010)$ & 95 (5) \\
\hline 21 & 5 & $250(11) \dagger$ & $64(3)+\S$ & $40(5)+\S$ & $0.116(0.004)+\S$ & $0.223(0.012) \S$ & 96 (5) \\
\hline
\end{tabular}

* Sea level controls. $\nmid p<0.05$ by comparison with controls. $\S p<0.05$ by comparison with the corresponding value in the Hilltop or $\overparen{D}$ Madison group.

Bw, body weight; RVPP, right ventricular peak systolic pressure; RV, right ventricular; $L V+S$, left ventricular plus septal. 


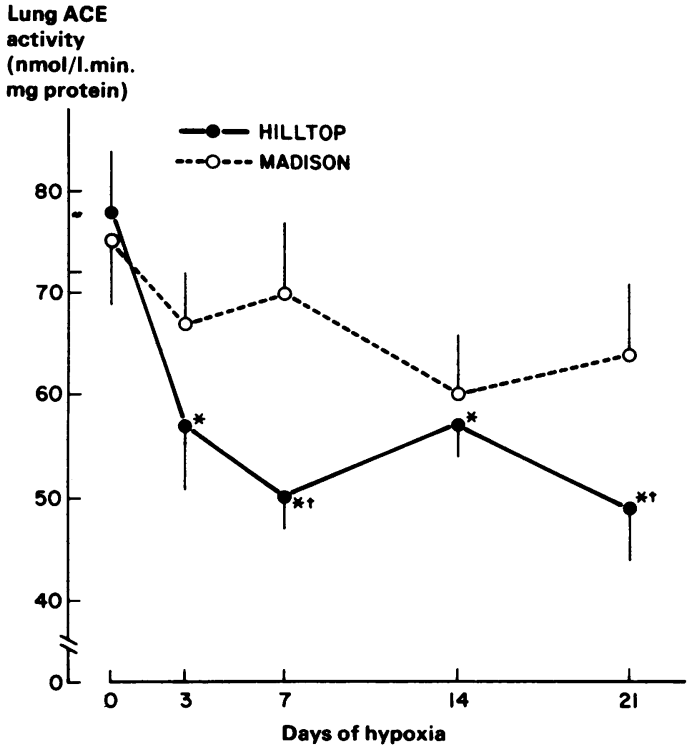

Fig 1 Mean (SEM) lung angiotensin converting enzyme ( $A C E$ ) activity for the two rat strains during hypoxia. ${ }^{*} p<$ 0.05 by comparison with the control value (day 0). $\dagger p<0.05$ by comparison with the simultaneous value in Madison rats. $n=12$ for each control group and 5 for each hypoxic group.

to those reported previously ${ }^{7}$ (table). $\mathrm{H}$ rats had a significantly greater packed cell volume than $M$ rats after seven and 21 days of hypobaria, a greater right ventricular weight index after 14 days, and a greater

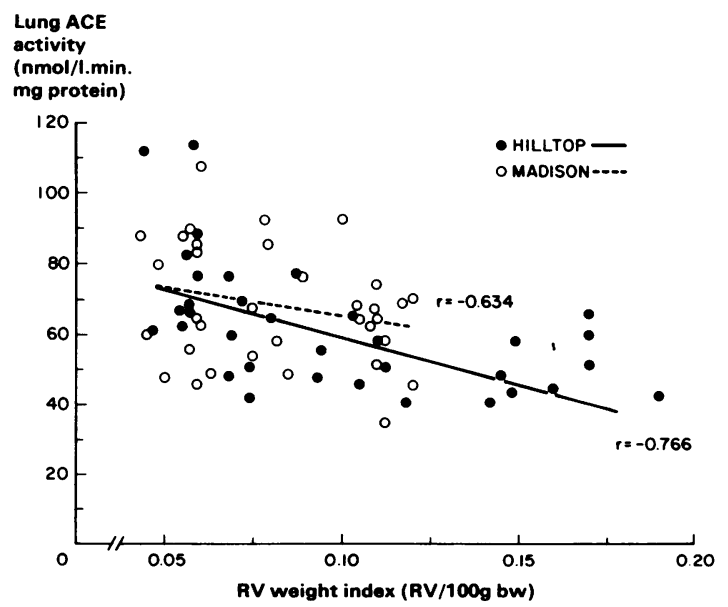

Fig 2 Individual data points and regression lines showing the relation between right ventricle $(R V)$ weight index and lung angiotensin converting enzyme (ACE) activity among control and chronically hypoxic rats. There was a significant inverse relationship between the two variables for both strains; the slopes of the regression lines did not differ significantly.

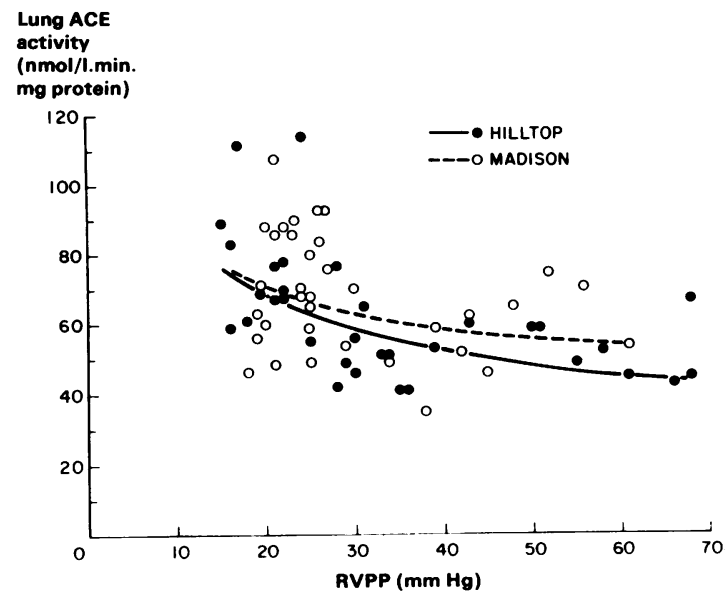

Fig 3 Hyperbolic curves and individual data points showing the relation between right ventricular peak systolic pressure (RVPP) and lung angiotensin converting enzyme ( $A C E$ ) activity in control and chronically hypoxic rats. The slopes of the curves did not differ significantly.

left ventricular plus septal weight index after 21 days.

Right ventricular peak systolic pressure was significantly greater at three days in the $\mathrm{H}$ rats and at seven days in the $M$ rats than in the control rats (table), and was significantly greater in $\mathrm{H}$ than in $\mathrm{M}$ rats from

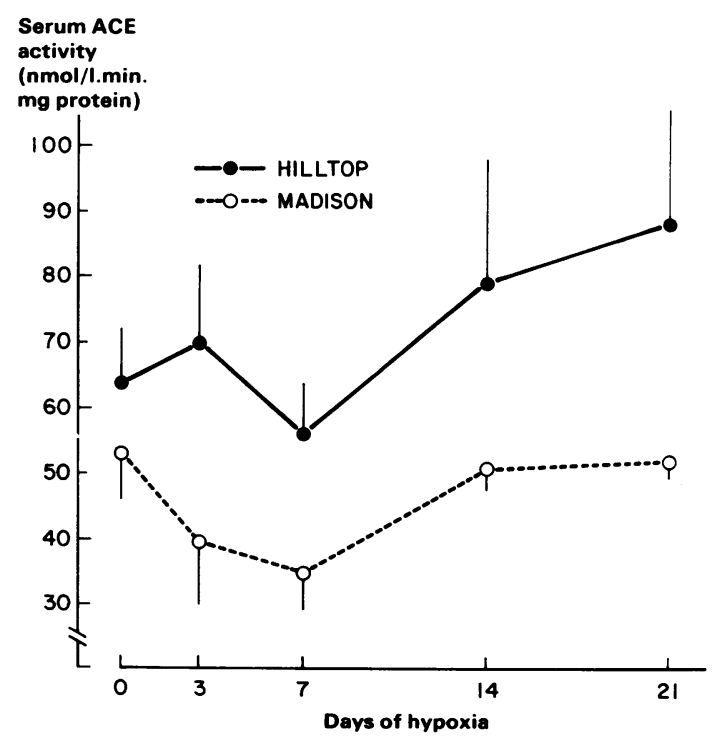

Fig 4 Mean (SEM) values for serum angiotensin converting enzyme ( $A C E$ ) activity during hypoxia in the two rat strains. Mean values were significantly greater in Hilltop than in Madison rats $(p<0.05)$ at each time point during hypoxia, but not at atmospheric pressure. $n=12$ for each control group and 5 for each hypoxic group. 
the seventh day of hypobaria. Protein concentrations in lung homogenates did not change during the course of the study.

Lung ACE activity decreased significantly from control levels within three days of the start of hypoxia in $\mathrm{H}$ rats (fig 1). A downward trend in lung ACE activity in $\mathbf{M}$ rats during exposure to hypoxia did not reach statistical significance.

Linear regression analysis of lung ACE activity and the right ventricular weight index showed significant inverse correlations for both $H(r=-0.766)$ and $M$ $(r=-0.634)$ rats (fig 2). Regression coefficients were not significantly different between the groups.

The relation between lung ACE activity and right ventricular peak systolic pressure was represented better by a hyperbolic than by a linear curve (fig 3 ). With standard curve fitting procedures the relation between lung ACE activity and right ventricular peak systolic pressure was expressed by the equation $\log y$ $=-0.202 \log x+2.11$ for $M$ rats and $\log y=-0.33$ $\log x+2 \cdot 27$ for $\mathrm{H}$ rats. The slopes of these curves were not significantly different.

Serum ACE activity displayed a tendency to increase in $\mathrm{H}$ rats during hypobaric exposure (fig 4), but differences from sea level ACE activity did not reach significance. In $M$ rats serum ACE activity remained more or less steady for the duration of the study. Serum ACE activities in $\mathrm{H}$ rats were significantly higher than those in $\mathbf{M}$ rats during hypobaric exposure.

\section{Discussion}

Our results show that lung ACE activity decreases more with chronic hypoxia in the $\mathrm{H}$ strain of SpragueDawley rat than in the $\mathbf{M}$ strain. The $\mathrm{H}$ strain also develops greater pulmonary hypertension in response to chronic hypoxia than the $M$ strain, supporting the hypothesis that depression of lung ACE activity in response to chronic hypoxia is related to the severity of the haemodynamic response. In an earlier study ${ }^{8}$ the two strains of rat had a similar degree of hypoxaemia after four weeks' exposure despite the differing cardiopulmonary responses. Differences in lung ACE activity therefore were probably not caused by differences in the amoung of hypoxaemia, although we did not repeat oxygen tension measurements in the present study.

We cannot entirely exclude certain alternative hypotheses to explain the greater depression of lung ACE activity in $\mathrm{H}$ rats. Lung ACE activity in the $\mathrm{H}$ strain may be more sensitive to hypoxia than the $M$ strain. Alternatively, some other strain related difference, such as difference in packed cell volume, may have contributed to the greater depression of lung ACE activity in the $\mathrm{H}$ rats.
The hypothesis that pulmonary haemodynamic $\stackrel{5}{+}$ responses determine the depression of lung $A C E^{\circ}$ activity is strengthened by the significant inverse $\frac{\bar{O}}{\bar{n}}$ correlations between lung ACE activity and right $\frac{\bar{\sigma}}{\sigma}$ ventricular weight index in both rat strains. Although $\varrho$ lung ACE activity in hypobaric $M$ rats did not decrease significantly below the level in normobaric $\vec{\circ}$ controls during the study, the similar inverse correla- tions between lung ACE activity and right ventricular $\vec{\omega}$ weight index in the two strains of rat suggest that lung ACE activity was responding in a similar manner to $\times$ increases in pulmonary arterial pressure. The degree of $\vec{\omega}$ pulmonary hypertension in the $M$ rats may not have 0 been sufficient to reduce lung ACE activity significantly.

The hyperbolic shape of the curves relating lung 9 ACE activity and right ventricular peak systolic $\vec{c}$ pressure is of interest. The shape may reflect acute ${ }^{\infty}$ reductions in pulmonary arterial pressure in response $\underset{\mathbb{\Phi}}{\stackrel{+}{+}}$ to anaesthesia or to the brief normobaric exposure 3 before pressure measurements were made. Pressures. may have been higher before these manipulations, which would have rendered the curve more linear. It is $\varnothing^{\circ}$ also possible that depression in lung ACE activity ${ }^{\circ}$ occurs during the initial rise in pulmonary arterialo pressure with little further change thereafter.

Our results concur with those of several othero studies. Keane et al ${ }^{\text {s }}$ observed a decrease in lung ACE activity in rats during chronic hypoxia and after $\stackrel{\mathbb{Q}}{\complement}$ injection of monocrotaline. The decreased lung $\mathrm{ACE} \overrightarrow{\vec{A}}$ activity correlated inversely with right ventricular 3 systolic pressure in both types of pulmonary hypertension. The same group ${ }^{6}$ noted a gradual recovery of lung ACE activity after the return of the hypoxic rats to normoxic conditions corresponding to the gradualo normalisation of right ventricular pressures. They considered this further evidence that haemodynamico responses rather than hypoxia caused the depression. of lung ACE activity.

The decrease in total lung ACE activity has been shown to correspond to measurable reductions in음 intravascular conversion of angiotensin $I$ to angioten- $\rightarrow$ sin II. Caldwell and Blatteis ${ }^{4}$ and Jin et al ${ }^{15}$ showedo․ㅡ․ smaller pulmonary pressor responses to infused $\bar{N}$ angiotensin $I$ in intact rats after 14 days of hypoxia. than in normoxic controls, suggesting decreased con- $N$ version to angiotensin II. Jackson et al ${ }^{16}$ measured the ${ }_{\mathcal{E}}^{N}$ generation of angiotensin II from angiotensin I injec-O ted into isolated rat lungs, and found it to beo significantly less in chronically hypoxic rats than in normoxic controls. The reduction was unaffected by? changes in mean transit time, and the authors sugges- $\square$ ted that it was related to alterations at the endothelialo membrane level.

Some investigators have reported results conflicting $\stackrel{\vec{\Phi}}{\mathbb{Q}}$ with these. Molteni et $a l^{3}$ found an increase in lungo 
ACE activity in chronically hypoxic mice, but species differences or differences in the assay technique may explain the difference from our results. Jackson et al ${ }^{16}$ noted no differences in total lung ACE activity of lung homogenates from normoxic and chronically hypoxic rats, but assays were performed after lung isolation and perfusion.

The mechanism and physiological significance of decreased lung ACE activity after exposure of rats to chronic hypoxia is unknown. Increased intravascular pressure may decrease synthesis or increase degradation of ACE, or promote release of ACE from the endothelial surface into the blood. The fact that serum ACE activity was greater in the $H$ than in the $M$ rats is consistent with the latter possibility. The resulting reduction in lung ACE activity could be compensatory, diminishing the conversion of angiotensin I to angiotensin II and thereby attenuating the increase in pressure. Our findings suggest that lung ACE does not contribute directly to the development of chronic hypoxic pulmonary hypertension, as postulated by some investigators. ${ }^{2}$ The reduction in the activity of angiotensin converting enzyme in response to raised pulmonary artery pressure may help modulate the hypertensive response.

NSH was supported by a National Heart, Lung and Blood Institute clinical investigator award and a Parker B Francis Foundation fellowship award.

\section{References}

1 Ryan JW, Ryan US. Pulmonary endothelial cells. Fed Proc 1977;36:2683-91.

2 Zakheim RM, Mattioli L, Molteni A, Mullis KB, Bartley J. Prevention of pulmonary vascular changes of chronic alveolar hypoxia by inhibition of angiotensin I converting enzyme in the rat. Lab Invest 1975;33:57-61.
3 Molteni A, Zakheim RM, Mullis KB, Mattioli L. The effect of chronic alveolar hypoxia on lung and serum angiotensin I converting enzyme activity. Proc Soc Exp Biol Med 1974;147:263-4.

4 Caldwell RW, Blatteis CM. Effect of chronic hypoxia on angiotensin-induced pulmonary vasoconstriction and converting enzyme activity in the rat. Proc Soc Exp Biol Med 1983;172:346-50.

5 Keane PM, Kay JM, Suyama KL, Gauthier D, Andrew K. Lung angiotensin converting enzyme activity in rats with pulmonary hypertension. Thorax 1982;37: 198-204.

6 Kay JM, Keane PM, Suyama KL, Gauthier D. Lung angiotensin converting enzyme activity in chronically hypoxic rats. Thorax 1985;40:587-91.

$7 \mathrm{Ou}$ LC, Smith RP. Probable strain differences of rats in susceptibilities and cardiopulmonary responses to chronic hypoxia. Respir Physiol 1983;53:367-77.

$8 \mathrm{Ou}$ LC, Hill NS, Tenney SM. Ventilatory responses and blood gases in susceptible and resistant rats to high altitude. Respir Physiol 1984;58:161-70.

9 Fulton RM, Hutchinson EC, Jones AM. Ventricular weight in cardiac hypertrophy. Br Heart $J$ 1952; 14:413-20.

10 Hill NS, Ou LC, Smith RP. Time course of cardiopulmonary responses to high altitude in susceptible and resistant rat strains. Respir Physiol 1987;70:241-9.

11 Lanzillo JJ, Fanburg BL. Membrane-bound angiotensin converting enzyme from rat lung. $J$ Biol Chem 1974;249:2312-8.

12 Lowry OH, Rosebrough NJ, Farr AL, Randall RJ. Protein measurement with the Folin phenol reagent. J Biol Chem 1951;193:265-75.

13 Steel RCD, Torrie JH. Principles and procedures of statistics. New York: McGraw-Hill, 1960:110-1.

14 Lewis D. Quantitative methods. New York: McGrawHill, 1960;51-80.

15 Jin H, Oparil S, Ann HS, Yang R, Jackson RM. Hypoxiaunduced inhibition of converting enzyme activity: role in vascular regulation. J Appl Physiol 1987;63:1012-8.

16 Jackson RM, Narkatis AJ, Oparil S. Impaired pulmonary conversion of angiotensin I to angiotensin II in rats exposed to chronic hypoxia. J Appl Physiol 1986;60:1121-7. 